\title{
LO QUE Sí FUNCIONA EN EDUCACIÓN
}

\author{
Roberto Barrientos Mollo \\ Coordinador general de Comunidades de Aprendizaje
}

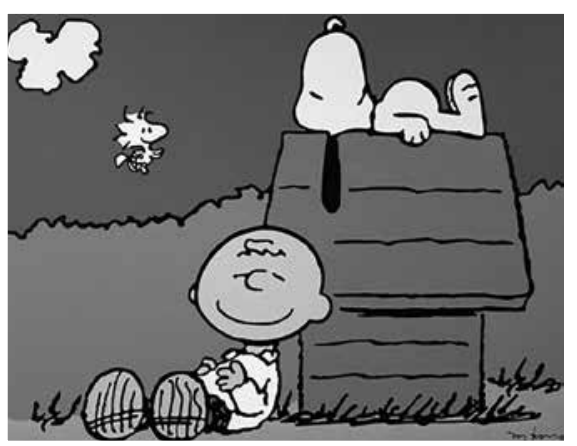

En un episodio de la serie "Charlie Brown", el protagonista les apostó a sus amigos que le enseñaría a hablar a Snoopy, su perro. Luego de una semana, ellos se acercaron y le reclamaron la apuesta argumentando que la mascota no había logrado hablar. La respuesta de Charlie fue bastante asertiva y clara: "Yo no dije que iba a aprender a hablar yo solo aposté a que le iba a enseñar a hablar, y eso es lo que hice". Esta historia grafica el dilema de la educación del siglo XX. Hemos generado un sistema, una infraestructura y un andamiaje que garantiza la enseñanza, pero no el aprendizaje. No es un secreto para nadie que cuando hemos cumplido el programa curricular, o creído cumplir, cada vez más estudiantes no logran lo esperado. Como dicen Dufour, Eaker y Karhanek en el siglo XX la constante ha sido el tiempo y el apoyo que el sistema educativo ofrecía a cada estudiante y en el siglo XXI la constante será el garantizar el aprendizaje de calidad en todos los estudiantes (20 I0). Es decir, en el siglo XX el tiempo que se ha ofrecido para aprender es el mismo para todos, bajo el supuesto de que todos los alumnos son iguales y aprenden en el mismo tiempo, y los aprendizajes han sido lo variable (figura I). Por cada I, I 00 horas de enseñanza en el sistema educativo en primaria solo $26,6 \%$ de estudiantes logran lo esperado en matemática, como muestran los resultados de la reciente evaluación de estudiantes de segundo grado del año 20 I 5. Igual número de apoyo para cada estudiante y una amplia variación en lo logros de aprendizajes. Lo que debe ocurrir en el siglo XXI es que la constante sea que el I $100 \%$ de los estudiantes aprendan versus el 26,6\% actual, pero para eso se necesita un cambio estructural acerca del tiempo y apoyo que se ofrece a cada estudiante. Tenemos que cambiar de chip y entender que quizá algunos estudiantes necesitarán I,800 horas y otros tipos de apoyo para que se pueda llegar a ese 100\% de logro. Ello implica repensar el sistema y trabajar sobre las prácticas más efectivas en educación ¿Cómo lograrlo? Allí entra la creatividad e innovación de todos aquellos interesados en que los estudiantes realmente aprendan. 


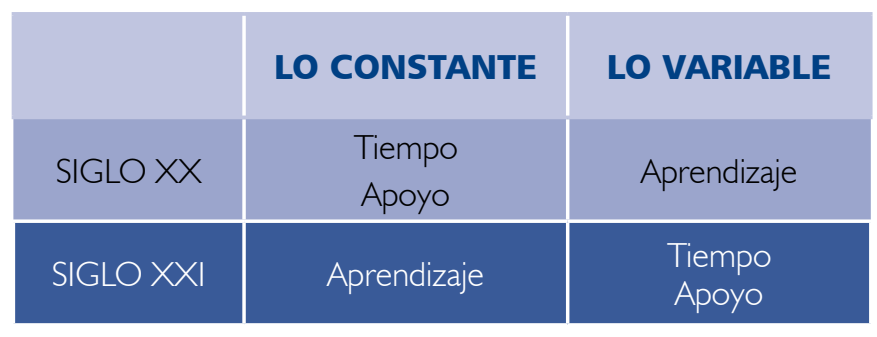

Figura l. Los cambios en la enseñanza y el aprendizaje. Traducción personal de DuFour, Eaker, \& Karhanek, (20।0).

Es por ello que una de las acciones que se debe realizar para atender mejor a los estudiantes es conocer, reflexionar y poner en práctica las mejores estrategias que existan en educación. Necesitamos saber cuáles son las acciones del docente que realmente ayudan al aprendizaje de los estudiantes, para poder hacer que el tiempo invertido en la enseñanza no sea solo de cumplimiento sino de efectividad pedagógica. En este texto quisiera mostrar lo que la investigación nos dice que sí funciona para mejorar aprendizajes.

Muchas veces el aprendizaje es borroso para nosotros y tenemos pocas maneras de ver lo que pasa en la mente y corazón de los estudiantes. Tampoco es fácil correlacionar el grado de contribución real de una estrategia determinada para lograr aprendizajes en los estudiantes. Asumimos mucho en educación y muchos de esos supuestos no tienen un fundamento claro. $\mathrm{O}$, lo que también suele ocurrir, es que se hacen generalizaciones de un estudio pequeño que abarcó una muestra no representativa que no permite generalizar. Ello también hace que la labor docente se realice con poca solidez en el aula. En este punto el aporte de los metanálisis es clave. El investigador neozelandés John Hattie le ha dedicado más de treinta años de su vida a tratar de desentrañar los misterios del aprendizaje. Hattie es investigador de la de la universidad de Auckland en Nueva Zelanda y realizó durante 15 años más de 800 metanálisis junto con una revisión de 50 mil artículos de investigación relacionados con el logro en los aprendizajes, utilizando una data que involucraba a más de 240 millones de estudiantes. Su esfuerzo buscó desvelar los elementos de la práctica docente efectiva, aquella que logra "hacer visible la enseñanza para los estudiantes, y el aprendizaje para los profesores" (J. Hattie, 20 I2, p. 25).

\section{Los metanálisis}

A manera de paréntesis es necesario explicar qué es un metanálisis. Un metanálisis es un ejercicio estadístico realizado con la data de decenas o cientos de investigaciones sobre un determinado tema: tareas escolares, enfoques de lectura o matemáticas, 
etc. El gran aporte de los metanálisis es que se realiza una síntesis estadística de mucha información lo que ofrece una mayor solidez para realizar generalizaciones. Si bien la generalización siempre es un aspecto que se debe realizar con mucho cuidado, los descubrimientos realizados por los metanálisis nos ofrecen indicios sobre dónde buscar la buena práctica educativa (Glass, MacGaw, \& Smith, 1984). Dichas síntesis trabajan con un índice que se llama tamaño del efecto (sise effect). El tamaño del efecto muestra el grado de incidencia o contribución de una determinada variable sobre el logro de aprendizajes. A partir de 0.4 se puede considerar como efectos positivos sobre aprendizaje. Los metanálisis han entrado al ámbito educativo relativamente hace poco. En el mundo de la salud y otros ámbitos se vienen usando hace un buen tiempo y es una herramienta útil para elaborar políticas y tomar decisiones.

\section{Las estrategias con mayor efecto en los aprendizajes}

Son cinco las prácticas del docente en el aula que los cientos de metanálisis mostraron que generan mayores logros de aprendizaje para todos los estudiantes: la enseñanza recíproca, la retroalimentación, la autoverbalización y el autocuestionamiento, las estrategias metacognitivas y, por último, la resolución de problemas (J. Hattie, 2008). En la tabla I se presenta cada una de ellas con su respectivo tamaño del efecto.

Tabla I. Estrategias de enseñanza con mayor efecto en los logros de aprendizaje

\begin{tabular}{|l|c|}
\hline \multicolumn{1}{c|}{ Estrategia } & Tamaño del efecto \\
\hline Enseñanza recíproca & 0.73 \\
\hline Retroalimentación & 0.74 \\
\hline Auto-verbalizar y auto-cuestionarse & 0.64 \\
\hline Las estrategias metacognitivas & 0.69 \\
\hline Resolución de problemas & 0.61 \\
\hline
\end{tabular}

Elaboración propia a partir de Hattie, 2010.

A continuación se desarrollan las principales.

\subsection{La Enseñanza recíproca}

La Enseñanza recíproca tiene un tamaño del efecto bastante alto, 0.76. En esta estrategia los docentes permiten que los estudiantes aprendan entre ellos y hagan uso del auto-aprendizaje (figura 2). Éstos asumen un rol de tutor para sus compañeros. Para el análisis de esta variable se realizó una síntesis de 38 estudios sobre el tema. 

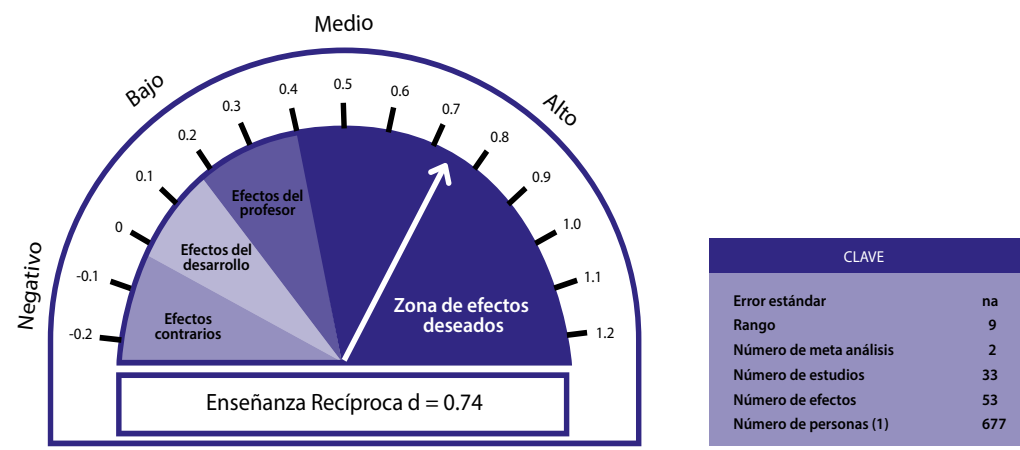

Figura 2. Tamaño del efecto de la enseñanza recíproca, elaboración propia a partir de Hattie (2008).

Diversos países con la evidencia abundante a favor de la enseñanza recíproca han focalizado sus esfuerzo de capacitación docente en el dominio efectivo de dicha estrategia. En Ontario (Canadá) ya hace varios años se viene capacitando a los docentes en la enseñanza recíproca. Sin embargo en otros países aún no se utiliza y difunde, a pesar de los grandes aportes para el bien de los estudiantes.

\subsection{La retroalimentación}

La segunda práctica con mayores efectos en logros de aprendizaje es la retroalimentación o feedback, con un 0.73 de tamaño del efecto. Consiste en ofrecer respuestas específicas al trabajo de los estudiantes. Es decir se realiza una evaluación para y como aprendizaje (Sharratt \& Fullan, 20I2). El propósito de la retroalimentación es "reducir discrepancias entre las actuales comprensiones de los estudiantes, el desempeño y un objetivo" (John Hattie \& Timperley, 2007, p. 86). O como dice Valerie Shute es "la información que se comunica al aprendiz que busca modificar su o conducta o pensamiento con el objetivo de mejorar el aprendizaje (2008, p. 157).

Marzano y Heflebower realizaron una investigación para medir el tamaño del efecto del feedback tomando en cuenta en número de veces que se realizaba y descubrieron algunos aspectos interesantes (20 I0). En la tabla I se puede observar que a mayor número de retroalimentaciones realizadas por el docente en un periodo de 15 semanas mayor era el tamaño del efecto para logros de aprendizaje. 
Tabla I. Tamaño del efecto del feedback a lo largo de 15 semanas.

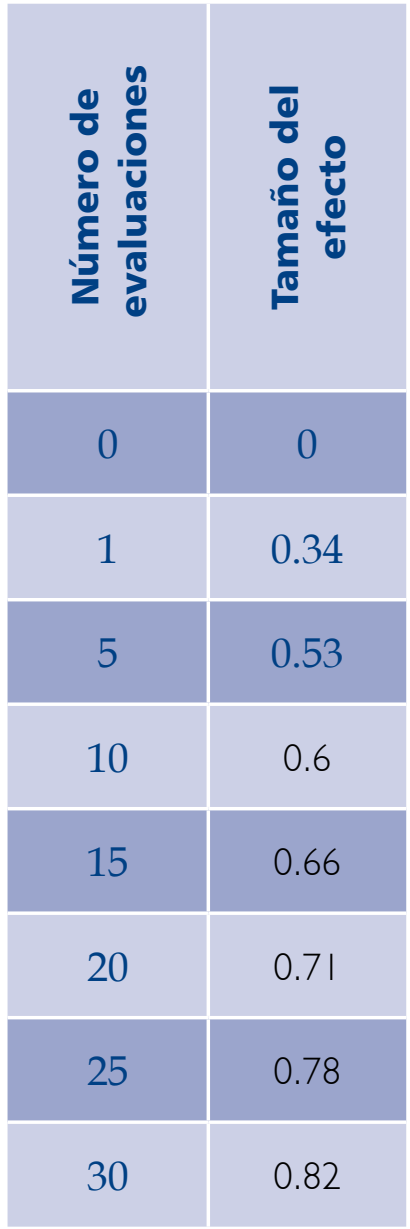

Traducción personal de Marzano y Hefleboweer, 2010.

Se podría deducir de esta información que realizar muchas evaluaciones a los estudiantes es positivo. Sin embargo lo más interesante viene después, cuando se realizó una síntesis de diversos estudios (Ver tabla 2). Esta síntesis mostró que no siempre realizar evaluaciones continuas es bueno. Muchas veces puede tener efectos contrarios a lo esperado. En la tabla 2 se puede observar que algunas veces no solo no hay efecto en el progreso sino que la evaluación misma resulta contraproducente. Es el caso de los estudios 4 y $1 \mathrm{I}$ de la tabla 2, en los que realizar evaluaciones continuas del progreso de los estudiantes hizo que empeoraran. 
Tabla 2. Estudios de seguimiento del progreso de los estudiantes.

\begin{tabular}{|c|c|}
\hline $\begin{array}{l}\frac{0}{0} \\
\frac{0}{2} \\
\frac{n}{4} \\
\text { th }\end{array}$ & 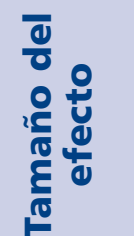 \\
\hline 1 & 2.44 \\
\hline 2 & 3.66 \\
\hline 3 & -1.50 \\
\hline 4 & -0.39 \\
\hline 5 & 0.75 \\
\hline 6 & 1.00 \\
\hline 7 & 0.07 \\
\hline 8 & 1.68 \\
\hline 9 & 0.07 \\
\hline 10 & 1.20 \\
\hline 11 & -0.32 \\
\hline 12 & 0.43 \\
\hline 13 & 0.84 \\
\hline 14 & 0.63 \\
\hline Promedio & 0.92 \\
\hline
\end{tabular}

Traducción personal de Marzano y Hefleboweer, 2010.

Por esta razón es bueno ser cautos con el uso de la evidencia. Lo que podemos concluir es que no cualquier evaluación es positiva para la mejora, además realizar evaluaciones sumativas semanales no siempre es bueno para que los estudiantes aprendan. La mejor retroalimentaciones es la descriptiva. La misma que requiere mucho entrenamiento y trabajo en equipo para llegar a dominarla. Otro vacío más en muchas reformas educativas en nuestro país. Revisemos las estrategias de capacitación que se han venido realizando hasta ahora y los contenidos de la estrategia de acompañamiento al docente para ver cuánto se ha incidido en el dominio del feedback descriptivo y la enseñanza recíproca como elementos centrales para mejorar los logros de aprendizaje. 


\subsection{Las estrategias metacognitivas}

Las otras dos estrategias más potentes son el enseñar a los alumnos a auto-verbalizar. Consiste en ofrecer a los alumnos espacios para que verbalicen lo que tienen que realizar y cómo lo realizarán. Esta estrategia tenía un 0.64 de tamaño del efecto y se realizaron 3 metanálisis de 113 estudios. Esta estrategia se puede enmarcar dentro de las estrategias para favorecer la metacognición. Las estrategias metacognitivas tienen un tamaño del efecto de 0.69 .

\subsection{La resolución de problemas}

Por último, la resolución de problemas es otra de las estrategias con un alto impacto en la mejora de logros de aprendizaje con un 0.61 de tamaño del efecto. Para la misma se realizaron 6 metanálisis de 221 estudios.

Como se ha afirmado, la información presentada debería ser un insumo para la definición de las políticas educativas. Asimismo, deberían ser el marco de referencia para la identificación, sistematización y difusión de las buenas prácticas que vienen ocurriendo en el salón de clases. Las diversas iniciativas de búsqueda de buenas prácticas nacidas de diversas direcciones del Ministerio de Educación (Educación Básica Especial, Dirección de Desarrollo Docente, Educación Superior Tecnológica, etc.), de las direcciones regionales, de las unidades de gestión educativa local y hasta de organizaciones no gubernamentales son loables y bienvenidas. En primer lugar porque la teoría del cambio que está detrás es que es necesario ir a las bases, al profesor de a pie, para ver lo que funciona en educación, lo cual es positivo. En segundo lugar porque es un reconocimiento a la sabiduría docente gestada en el día a día. Sin embargo se puede llegar a tener un conjunto de estrategias y acciones muy llamativas, pero nada más, con poca capacidad de réplica y sin una medición clara del impacto real en los resultados de los estudiantes. Pudiendo simplemente llegar a ser algo folclórico. Es por ello que es necesario encontrar una armonía entre lo que se llama Wisdom of crowd o sabiduría de las masas junto con los aportes de la investigación (Briskin \& Erickson, 2009). Por lo que una estrategia adecuada es, como se ha mencionado, tener un marco de referencia basado en las mejores síntesis de investigaciones y con ese marco empezar a recoger las mejores prácticas de los docentes en aula.

El trabajo de metanálisis realizado por Hattie nos ofrece, entonces, un insumo útil para saber lo que sí funciona en educación, sin embargo también nos ayuda a saber sobre lo que no funciona en educación (2010). A continuación veamos solo un ejemplo, el caso de las tareas para la casa. 


\section{Las tareas para la casa}

Esta estrategia se viene utilizando hace muchos años y se da por sentado sus efectos positivos para lograr aprendizajes. Es más, muchos padres juzgan la calidad de una escuela por la cantidad de tareas para la casa que dejan a los estudiantes. Cooper que ha investigado las tareas escolares por mucho tiempo afirma que el efecto es el doble en secundaria que en primaria (1989). Los efectos positivos de las tareas para la casa tenía una relación negativa con la duración de la misma. Es decir, mientras más tiempo requería de los estudiantes menores efectos de aprendizaje se lograba. Sin embargo es necesario preguntarse si las tareas para la casa ayudan realmente a mejorar aprendizajes. Allí entra la investigación para darnos una mano. El tamaño del efecto de las mismas es de 0.29 , es decir tiene un efecto bastante bajo para lograr aprendizajes (figura 3).

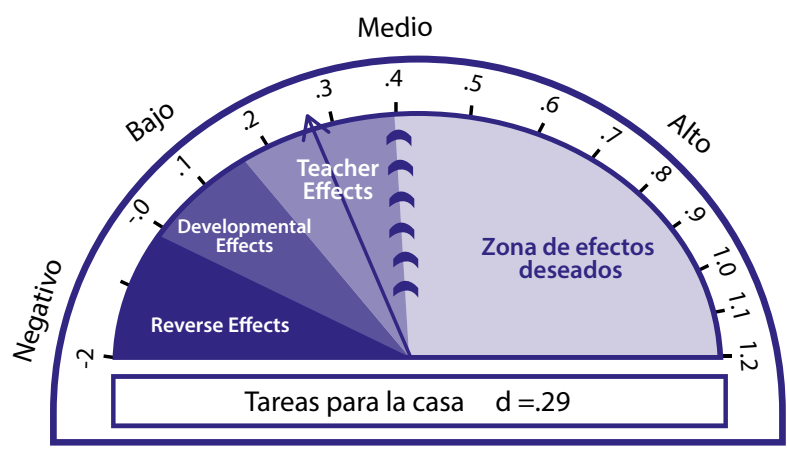

Figura 3. Tamaño del efecto de las tareas para la casa. Tomado de Hattie (2012)

Como se puede ver no todo lo que se hace en la actualidad en el sistema educativo ayuda a mejorar los aprendizajes.

\section{Factores extra e intraescolares que impactan más en los logros de aprendizaje}

El análisis realizado por Hattie tomó en cuenta diversas dimensiones de la vida escolar: Factores a nivel de estudiantes, factores del hogar, características de la escuelas, características del docente, enfoques curriculares y por últimos estrategias del trabajo docente (2010). En la figura 3 se presenta una síntesis de aquellas variables que tienen mayor impacto en la mejora de aprendizajes. 


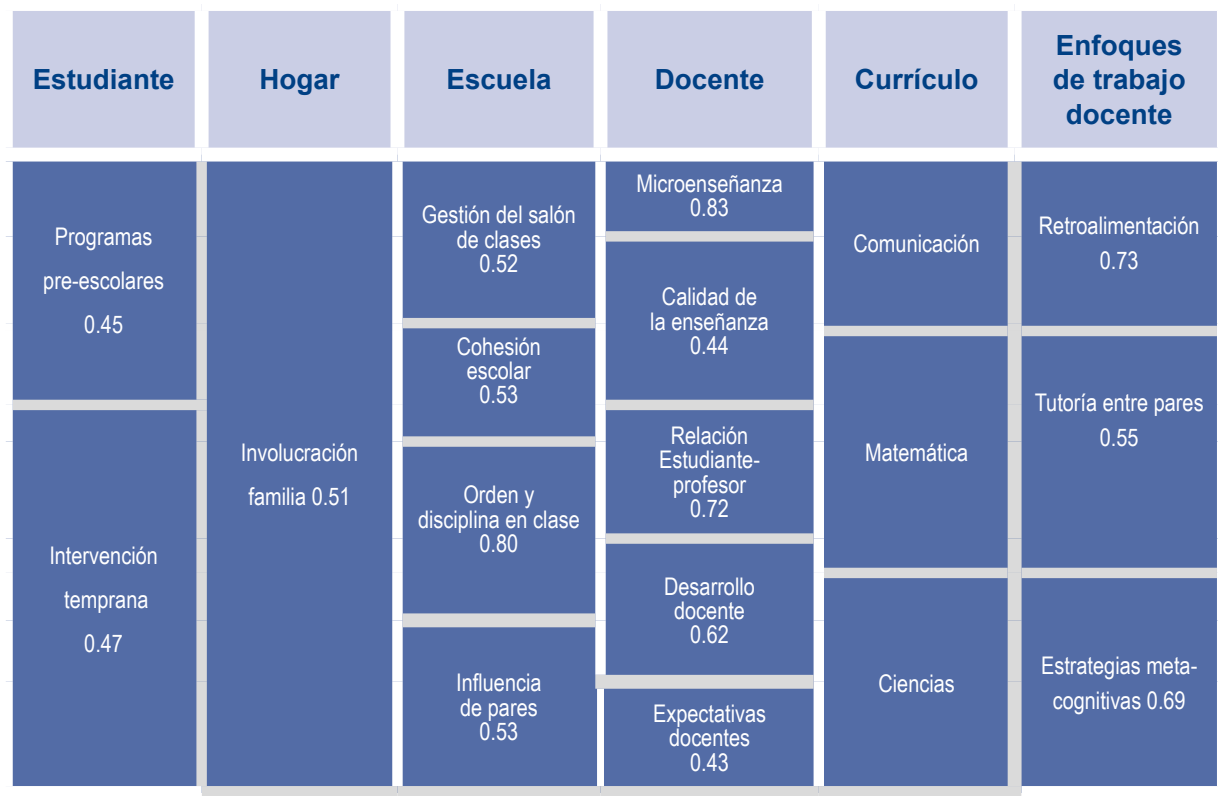

Figura 3. Tamaño del efecto de los principales factores que influyen en los aprendizajes según Hattie.

\section{Conclusiones}

El profesional de la educación necesita las mejores herramientas existentes para hacer bien su trabajo. En ese sentido, el aporte de la investigación por medio de los metanálisis resulta una herramienta importante. Queda en manos de los educadores y tomadores de decisión de órganos intermedios y centrales utilizar de la mejor manera esta información. El profesional reflexivo es aquel que sabe utilizarlo y sopesarlo adecuadamente para el logro de sus metas (Schön, 1988).

La información que se ha presentado debería ser un insumo para los directores de escuelas, que son los encargados de la formación continua de los docentes. Para los especialistas y directores de UGEL, que deben liderar el desarrollo de capacidades en sus respectivas zonas. Para los gobiernos regionales que están empezando a priorizar la educación, pero muchas veces, sin mala intención, invierten en lo que no sirve o no tiene alto impacto en la mejora de los aprendizajes. Para las empresas que tienen mucho intereses en ayudar en educación, pero no saben en qué ni cómo invertir. Por último, esta información es un insumo para los funcionarios del Ministerio de Educación, tanto en la Dirección de Educación Básica Regular como en la Dirección General de Desarrollo Docentes. Puesto que ambos son las dos patas de una misma mesa. Uno encargado del currículo y del cómo se debe enseñar y el otro vela por la formación continua de los docentes. 


\section{Referencias}

Briskin, A., \& Erickson, S. (2009). The power of collective wisdom: And the trap of collective folly: Berrett-Koehler Publishers.

Cooper, H. ( 1 989). Homework versus no-treatment: Longman.

DuFour, R., Eaker, R., \& Karhanek, G. (2010). Raising the bar and closing the gap: Whatever it takes: Solution Tree Press.

Glass, G. V., MacGaw, B., \& Smith, M. L. (1984). Meta-analysis in social research: Sage Beverly Hills, CA.

Hattie, J. (2008). Visible learning: A synthesis of over 800 meta-analyses relating to achievement: Routledge.

Hattie, J. (2012). Visible Learning for Teachers: maximizing impact on learning: Routledge.

Hattie, J., \& Timperley, H. (2007). The power of feedback. Review of Educational Research, 77(I), 81-112.

Marzano, R. J., \& Heflebower, T. (2010). Formative assessment \& standards-based grading: Solution Tree.

Schön, D. A. (1988). El profesional reflexivo: como piensan los profesionales cuando actúan: Paidos, Barcelona: P. imprenta.

Sharratt, L., \& Fullan, M. (20I2). Putting FACES on the Data: What Great Leaders Do! : Corwin Press.

Shute, V. J. (2008). Focus on formative feedback. Review of Educational Research, $78(1), 153-189$. 